\title{
Convolutions of Harmonic Functions with Certain Dilatations
}

\author{
Om P. Ahuja and Jay M. Jahangiri \\ Mathematical Sciences, Kent State University, Burton, OH 44021-9500, USA \\ Correspondence should be addressed to Jay M. Jahangiri; jjahangi@kent.edu
}

Received 1 October 2017; Accepted 13 November 2017; Published 29 November 2017

Academic Editor: Teodor Bulboaca

Copyright (C) 2017 Om P. Ahuja and Jay M. Jahangiri. This is an open access article distributed under the Creative Commons Attribution License, which permits unrestricted use, distribution, and reproduction in any medium, provided the original work is properly cited.

\begin{abstract}
The convolution of harmonic functions, unlike the analytic case, proved to be very challenging. In this paper, we introduce dilatation conditions that guarantee the convolution of two harmonic functions to be locally one-to-one, sense-preserving, and close-toconvex harmonic in the unit disk.
\end{abstract}

\section{Introduction}

Let $\mathscr{A}$ denote the class of functions that are analytic in the open unit disc $\mathbb{D}:=\{z \in \mathbb{C}:|z|<1\}$ and let $\mathscr{A}^{\prime}$ be the subclass of $\mathscr{A}$ consisting of functions $h$ with the normalization $h(0)=h^{\prime}(0)-1=0$. We let $\mathscr{K}(\alpha)$ denote the class of functions $h \in \mathscr{A}^{\prime}$ so that

$$
\operatorname{Re}\left\{1+z \frac{h^{\prime \prime}(z)}{h^{\prime}(z)}\right\}>\alpha ; \quad-\frac{1}{2} \leq \alpha ; z \in \mathbb{D} .
$$

Consider the family of complex-valued harmonic functions $f=u+i v$ defined in $\mathbb{D}$, where $u$ and $v$ are real harmonic in $\mathbb{D}$. Such functions can be expressed as $f=h+\bar{g}$, where $h \in \mathscr{A}$ and $g \in \mathscr{A}$. Clunie and Sheil-Small in their remarkable paper [1] explored the functions of the form $f=h+\bar{g}$ that are locally one-to-one, sense-preserving, and harmonic in $\mathbb{D}$. By Lewy's Theorem (see [2] or [1]), a necessary and sufficient condition for the harmonic function $f=h+\bar{g}$ to be locally one-to-one and sense-preserving in $\mathbb{D}$ is that its Jacobian $J_{f}=\left|h^{\prime}\right|^{2}-\left|g^{\prime}\right|^{2}$ is positive or equivalently, if and only if $h^{\prime}(z) \neq 0$ in $\mathbb{D}$ and the second complex dilatation $\omega$ of $f$ satisfies $|\omega|=\left|g^{\prime} / h^{\prime}\right|<1$ in $\mathbb{D}$.

In an interesting article, Bshouty and Lyzzaik [3] proved the following.

Theorem 1. Let $f=h+\bar{g}$ be a harmonic mapping of $\mathbb{D}$, with $h^{\prime}(0) \neq 0$, that satisfies $g^{\prime}(z)=z h^{\prime}(z)$ and $h \in \mathscr{K}(-1 / 2)$ for all $z \in \mathbb{D}$. Then $f$ is a univalent close-to-convex mapping.
A simply connected proper subdomain of $\mathbb{C}$ is said to be close-to-convex if its complement in $\mathbb{C}$ is the union of closed half-lines with pairwise disjoint interiors. Consequently, a univalent analytic or harmonic function $f: \mathbb{D} \rightarrow \mathbb{C}$ is said to be close-to-convex if $f(\mathbb{D})$ is close-to-convex (e.g., see Clunie and Sheil-Small [1] or Bshouty and Lyzzaik [3]).

Ruscheweyh and Sheil-Small in a striking article [4] proved that the Hadamard product or convolution of two analytic convex functions is also convex analytic and that the convolution of an analytic convex function and an analytic close-to-convex function is close-to-convex analytic in the unit disk $\mathbb{D}$. Ironically, these results could not be extended to the harmonic case, since the convolution of harmonic functions, unlike the analytic case, proved to be very challenging. The purpose of the present paper is to introduce dilatation conditions that guarantee the convolution of two harmonic functions to be locally one-to-one, sense-preserving, and close-to-convex harmonic in the unit disk $\mathbb{D}$. In other words, we extend Theorem 1 to the convolution of two harmonic functions $f_{1}=h_{1}+\overline{g_{1}}$ and $f_{2}=h_{2}+\overline{g_{2}}$ with certain dilatations, where $h_{1} * h_{2} \in \mathbb{K}(\alpha)$.

The operator $*$ stands for the convolution or Hadamard product of two power series $h_{1}(z)=\sum_{n=1}^{\infty} a_{n} z^{n}$ and $h_{2}(z)=$ $\sum_{n=1}^{\infty} b_{n} z^{n}$ given by $h_{1}(z) * h_{2}(z)=\left(h_{1} * h_{2}\right)(z)=\sum_{n=1}^{\infty} a_{n} b_{n} z^{n}$. Similarly, the convolution of two harmonic functions $f_{1}=$ $h_{1}+\overline{g_{1}}$ and $f_{2}=h_{2}+\overline{g_{2}}$ is given by $f_{1} * f_{2}=h_{1} * h_{2}+\overline{g_{1} * g_{2}}$.

In regard to the convolution of harmonic univalent functions, Clunie and Sheil-Small [1] proved the following. 
Theorem 2. If $\phi \in \mathscr{K}(0)$ and if $f$ is convex harmonic in $\mathbb{D}$, then their convolution $(\phi+\varepsilon \bar{\phi}) * f(|\varepsilon| \leq 1)$ is close-to-convex harmonic in $\mathbb{D}$.

A mapping $f: \mathbb{D} \rightarrow \mathbb{C}$ is called convex harmonic if $f(\mathbb{D})$ is a convex domain.

The convexity condition for the function $\phi$ in Theorem 2 cannot be compromised as it is demonstrated in the following.

Example 3. Set

$$
f(z)=h(z)+\overline{g(z)}=\frac{z-(1 / 2) z^{2}}{(1-z)^{2}}-\overline{\left(\frac{(1 / 2) z^{2}}{(1-z)^{2}}\right)}
$$

and consider the starlike analytic function $\phi(z)=z+z^{n} / n$; $n \geq 2$ in $\mathbb{D}$. Letting $\varepsilon=0$ in Theorem 2 , we observe that the harmonic convolution

$$
(\phi(z)+(0) \cdot \overline{\phi(z)}) *(h(z)+\overline{g(z)})=z+\frac{n+1}{2 n} z^{n}
$$

is not even univalent in $\mathbb{D}$.

In an attempt to investigate the possibilities of improving the required convexity condition for $\phi$, the authors in [5] proved the following.

Theorem 4. Let $\phi \in \mathscr{K}(0)$ and $h \in \mathscr{K}(0)$. Also let $w$ be a Schwarz function. Then the convolution function

$$
(\phi(z)+\varepsilon \overline{\phi(z)}) *\left(h(z)+\overline{\int_{0}^{z} w(t) h^{\prime}(t) d t}\right)
$$

$$
|\varepsilon|=1
$$

is close-to-convex harmonic in $\mathbb{D}$.

Theorem 4 for $\phi(z)=z /(1-z)$ and $\varepsilon=1$ yields a theorem given by Bshouty et al. ([6], Theorem 2). From what is said above, especially Example 3, one wonders if there are other conditions that guarantee the close-to-convexity of the convolution of two harmonic functions. In the following theorem, we find such conditions.

Theorem 5. Let $h_{1} \in \mathscr{A}^{\prime}$ and $h_{2} \in \mathscr{A}^{\prime}$ so that $h_{1} * h_{2} \in \mathscr{K}(\alpha)$, where $\mathscr{K}(\alpha)$ is given by inequality (1). If one of the following conditions hold

(i) $\alpha \geq-1 / 2 ; g_{1}(z)=z h_{1}(z)$ and $g_{2}^{\prime}(z)=z h_{2}^{\prime}(z)$,

(ii) $\alpha \geq 0 ; g_{1}^{\prime}(z)=z^{n} h_{1}^{\prime}(z)$ and $g_{2}^{\prime}(z)=z^{n} h_{2}^{\prime}(z)$, where $n \in \mathbb{N}$,

then the convolution function $F(z)=h_{1}(z) * h_{2}(z)+$ $\overline{g_{1}(z) * g_{2}(z)}$ is locally one-to-one, sense-preserving, and closeto-convex harmonic in $\mathbb{D}$.

Since the convolution of two convex analytic functions is also convex (see Ruscheweyh and Sheil-Small [4]), an obvious consequence of the above theorem would be as follows.
Corollary 6. Let $h_{1} \in \mathscr{K}(0)$ and $h_{2} \in \mathscr{K}(0)$ and set $g_{1}(z)=$ $z^{n} h_{1}^{\prime}(z)$ and $g_{2}^{\prime}(z)=z^{n} h_{2}^{\prime}(z)$. Then the convolution function $F(z)=h_{1}(z) * h_{2}(z)+\overline{g_{1}(z) * g_{2}(z)}$ is locally one-to-one, sensepreserving, and close-to-convex harmonic in $\mathbb{D}$.

\section{Preliminary Lemmas and Proof of Theorem 5}

To prove our Theorem 5, we shall need the following three lemmas, the first of which is a celebrated result by Clunie and Sheil-Small [1] and the second one is given by Kaplan [7]. The third lemma which is on subordination is a modification of a result given by Miller and Mocanu (e.g., see [8] Lemma 1 or [9]). For functions $p$ and $q$, where $p(0)=q(0)=0$, we write $p \prec q$ (i.e., $p$ is subordinate to $q$ ) if there exists an analytic function $\omega$ with $\omega(0)=0$ and $|\omega(z)|<1$ so that $p(z)=q(\omega(z))$ in $\mathbb{D}$.

Lemma 7. (i) If $g$ and $h$ are analytic in $\mathbb{D}$ so that $\left|g^{\prime}(0)\right|<$ $\left|h^{\prime}(0)\right|$ and if $h+\varepsilon g$ is close-to-convex analytic in $\mathbb{D}$ for each $\varepsilon(|\varepsilon|=1)$, then the function $f=h+\bar{g}$ is close-to-convex harmonic in $\mathbb{D}$.

(ii) If $h$ and $g$ are analytic in $\mathbb{D}$ so that $h \in \mathscr{K}(0)$ and if $f=h+\bar{g}$ is locally univalent in $\mathbb{D}$, then the function $f=h+\bar{g}$ is close-to-convex harmonic in $\mathbb{D}$.

Lemma 8. A necessary and sufficient condition for the analytic function $h: \mathbb{D} \rightarrow \mathbb{C}$ to be close-to-convex is that $h^{\prime}$ is nonvanishing in $\mathbb{D}$ and

$$
\int_{\theta_{1}}^{\theta_{2}} \operatorname{Re}\left\{1+r e^{i \theta} \frac{h^{\prime \prime}\left(r e^{i \theta}\right)}{h^{\prime}\left(r e^{i \theta}\right)}\right\} d \theta>-\pi ;
$$

$$
\theta_{1}<\theta_{2}, 0<r<1 \text {. }
$$

Lemma 9. If $\Re p(z)>0$ and $q(z)$ is analytic in $\mathbb{D}$, then $q(z)+$ $z p(z) q^{\prime}(z) \prec z$ implies $q(z) \prec z$.

\section{Proof of Theorem 5.}

Proof of Part (i). The convolution function $F(z)=h_{1}(z) *$ $h_{2}(z)+\overline{g_{1}(z) * g_{2}(z)}=H(z)+\overline{G(z)}$ is locally univalent and sense-preserving since

$$
\begin{aligned}
\left|\frac{G^{\prime}(z)}{H^{\prime}(z)}\right| & =\left|\frac{(1 / z) g_{1}(z) * g_{2}^{\prime}(z)}{(1 / z) h_{1}(z) * h_{2}^{\prime}(z)}\right|=\left|\frac{g_{1}(z) * z g_{2}^{\prime}(z)}{h_{1}(z) * z h_{2}^{\prime}(z)}\right| \\
& =\left|\frac{z h_{1}(z) * z^{2} h_{2}^{\prime}(z)}{h_{1}(z) * z h_{2}^{\prime}(z)}\right|=|z|<1 .
\end{aligned}
$$

Obviously $\left|G^{\prime}(0)\right|<\left|H^{\prime}(0)\right|$; therefore, in view of Lemma 7, it suffices to prove that $T_{\lambda}(z)=H(z)-\lambda G(z)$ for $|\lambda|=1$ is close-to-convex analytic in $\mathbb{D}$.

We note that

$$
\begin{aligned}
T_{\lambda}^{\prime}(z) & =H^{\prime}(z)-\lambda G^{\prime}(z) \\
& =H^{\prime}(z)-\lambda\left((1 / z) g_{1}(z) * g_{2}^{\prime}(z)\right)
\end{aligned}
$$




$$
\begin{aligned}
& =H^{\prime}(z)-\lambda\left(h_{1}(z) * z h_{2}^{\prime}(z)\right) \\
& =H^{\prime}(z)-\lambda z\left((1 / z) h_{1}(z) * h_{2}^{\prime}(z)\right) \\
& =H^{\prime}(z)-\lambda z\left(H^{\prime}(z)\right)=(1-\lambda z) \cdot H^{\prime}(z),
\end{aligned}
$$

and $T_{\lambda}^{\prime \prime}(z)=-\lambda H^{\prime}(z)+(1-\lambda z) H^{\prime \prime}(z)$.

We also observe that $T_{\lambda}$ is nonvanishing in $\mathbb{D}$ since $H^{\prime}(0) \neq 0$. Therefore,

$$
\begin{aligned}
& \operatorname{Re}\left\{1+z \frac{T_{\lambda}^{\prime \prime}(z)}{T_{\lambda}^{\prime}(z)}\right\} \\
& =\operatorname{Re}\left\{1+z \frac{-\lambda H^{\prime}(z)+(1-\lambda z) H^{\prime \prime}(z)}{(1-\lambda z) H^{\prime}(z)}\right\} \\
& =\operatorname{Re}\left\{1+\frac{\lambda z}{\lambda z-1}+z \frac{H^{\prime \prime}(z)}{H^{\prime}\left(r e^{i \theta}\right)}\right\} .
\end{aligned}
$$

Now, by Lemma 8 and inequality (5) for $\theta_{1}<\theta_{2}<\theta_{1}+2 \pi$ and $0<r<1$, it suffices to show that

$$
\int_{\theta_{1}}^{\theta_{2}} \operatorname{Re}\left\{1+r e^{i \theta} \frac{T_{\lambda}^{\prime \prime}\left(r e^{i \theta}\right)}{T_{\lambda}^{\prime}\left(r e^{i \theta}\right)}\right\} d \theta>-\pi
$$

For $z \in \mathbb{D}$, one may verify (also see Bshouty and Lyzzaik [3] p. 770) that

$$
\operatorname{Re}\left\{\frac{z}{z-1}\right\}=\frac{1}{2}-\frac{1}{2} \frac{1-|z|^{2}}{|1-z|^{2}}
$$

For $z=r e^{i \theta}$, replacing $z$ by $\lambda z$ and letting $\zeta=\bar{\lambda} r$ yield

$$
\operatorname{Re}\left\{\frac{\lambda z}{\lambda z-1}\right\}=\frac{1}{2}-\frac{1}{2} \frac{1-|\zeta|^{2}}{\left|e^{i \theta}-\zeta\right|^{2}}=\frac{1}{2}-\frac{1}{2} P_{\zeta}(\theta)
$$

where $P_{\zeta}(\theta)$ is the Poisson Kernal. It then follows that

$$
\begin{gathered}
\int_{\theta_{1}}^{\theta_{2}} \operatorname{Re}\left\{\frac{\lambda r e^{i \theta}}{\lambda r e^{i \theta}-1}\right\} d \theta=\int_{\theta_{1}}^{\theta_{2}} \operatorname{Re}\left\{\frac{1}{2}-\frac{1}{2} \cdot P_{\zeta}(\theta)\right\} d \theta \\
=\frac{\theta_{2}-\theta_{1}}{2}-\frac{1}{2} \int_{\theta_{1}}^{\theta_{2}} P_{\zeta}(\theta) d \theta \\
>\frac{\theta_{2}-\theta_{1}}{2}-\frac{1}{2} \int_{0}^{2 \pi} P_{\zeta}(\theta) d \theta=\frac{\theta_{2}-\theta_{1}}{2}-\pi .
\end{gathered}
$$

On the other hand, since $H(z)=h_{1}(z) * h_{2}(z) \in \mathscr{K}(-1 / 2)$, we obtain

$$
\int_{\theta_{1}}^{\theta_{2}} \operatorname{Re}\left\{1+r e^{i \theta} \frac{H^{\prime \prime}\left(r e^{i \theta}\right)}{H^{\prime}\left(r e^{i \theta}\right)}\right\} d \theta>\frac{\theta_{1}-\theta_{2}}{2} .
$$

Therefore, in view of the required condition (9), we get

$$
\begin{gathered}
\int_{\theta_{1}}^{\theta_{2}} \operatorname{Re}\left\{1+r e^{i \theta} \frac{T_{\lambda}^{\prime \prime}\left(r e^{i \theta}\right)}{T_{\lambda}^{\prime}\left(r e^{i \theta}\right)}\right\} d \theta \\
=\int_{\theta_{1}}^{\theta_{2}} \operatorname{Re}\left(1+\frac{\lambda r e^{i \theta}}{\lambda r e^{i \theta}-1}\right) d \theta \\
\quad+\int_{\theta_{1}}^{\theta_{2}} \operatorname{Re}\left(r e^{i \theta} \frac{H^{\prime \prime}\left(r e^{i \theta}\right)}{H^{\prime}\left(r e^{i \theta}\right)}\right) d \theta \\
>\frac{\theta_{2}-\theta_{1}}{2}-\pi+\frac{\theta_{1}-\theta_{2}}{2}=-\pi .
\end{gathered}
$$

Proof of Part (ii). In view of Lemma 7, it suffices to show that the convolution function $F(z)=h_{1}(z) * h_{2}(z)+$ $\overline{g_{1}(z) * g_{2}(z)}=H(z)+\overline{G(z)}$ is locally univalent and sensepreserving in $\mathbb{D}$. In other words, we need to show that

$$
\left|\frac{G^{\prime}(z)}{H^{\prime}(z)}\right|=\left|\frac{\left[g_{1}(z) * g_{2}(z)\right]^{\prime}}{\left[h_{1}(z) * h_{2}(z)\right]^{\prime}}\right|<1 .
$$

Using the Hadamard product properties of power series, we have

$$
\begin{aligned}
1 & >\left|z^{n}\right|=\left|\frac{z^{n}\left(h_{1}^{\prime} * h_{2}^{\prime}\right)}{h_{1}^{\prime} * h_{2}^{\prime}}\right|=\left|\frac{g_{1}^{\prime} * g_{2}^{\prime}}{h_{1}^{\prime} * h_{2}^{\prime}}\right|=\left|\frac{\left[g_{1} * z g_{2}^{\prime}\right]^{\prime}}{\left[h_{1} * z h_{2}^{\prime}\right]^{\prime}}\right|=\left|\frac{\left[z\left((1 / z) g_{1} * g_{2}^{\prime}\right)\right]^{\prime}}{\left[z\left((1 / z) h_{1} * h_{2}^{\prime}\right)\right]^{\prime}}\right|=\left|\frac{\left[z\left(g_{1} * g_{2}\right)^{\prime}\right]^{\prime}}{\left[z\left(h_{1} * h_{2}\right)^{\prime}\right]^{\prime}}\right| \\
& =\left|\frac{\left(g_{1} * g_{2}\right)^{\prime}+z\left(g_{1} * g_{2}\right)^{\prime \prime}}{\left[z\left(h_{1} * h_{2}\right)^{\prime}\right]^{\prime}}\right|=\left|\frac{\left(g_{1} * g_{2}\right)^{\prime}\left(h_{1} * h_{2}\right)^{\prime}+z\left(g_{1} * g_{2}\right)^{\prime \prime}\left(h_{1} * h_{2}\right)^{\prime}}{\left(h_{1} * h_{2}\right)^{\prime}\left[z\left(h_{1} * h_{2}\right)^{\prime}\right]^{\prime}}\right| \\
& =\left|\frac{\left(g_{1} * g_{2}\right)^{\prime}\left[\left(h_{1} * h_{2}\right)^{\prime}+z\left(h_{1} * h_{2}\right)^{\prime \prime}\right]+z\left(g_{1} * g_{2}\right)^{\prime \prime}\left(h_{1} * h_{2}\right)^{\prime}-z\left(g_{1} * g_{2}\right)^{\prime}\left(h_{1} * h_{2}\right)^{\prime \prime}}{\left(h_{1} * h_{2}\right)^{\prime}\left[z\left(h_{1} * h_{2}\right)^{\prime}\right]^{\prime}}\right| \\
& =\left|\frac{\left(g_{1} * g_{2}\right)^{\prime}\left[z\left(h_{1} * h_{2}\right)^{\prime}\right]^{\prime}+z\left(g_{1} * g_{2}\right)^{\prime \prime}\left(h_{1} * h_{2}\right)^{\prime}-z\left(g_{1} * g_{2}\right)^{\prime}\left(h_{1} * h_{2}\right)^{\prime \prime}}{\left(h_{1} * h_{2}\right)^{\prime}\left[z\left(h_{1} * h_{2}\right)^{\prime}\right]^{\prime}}\right|
\end{aligned}
$$




$$
\begin{aligned}
& =\left|\frac{\left(g_{1} * g_{2}\right)^{\prime}}{\left(h_{1} * h_{2}\right)^{\prime}}+\frac{z}{\left[z\left(h_{1} * h_{2}\right)^{\prime}\right]^{\prime}}\left(\frac{\left(g_{1} * g_{2}\right)^{\prime \prime}\left(h_{1} * h_{2}\right)^{\prime}-\left(g_{1} * g_{2}\right)^{\prime}\left(h_{1} * h_{2}\right)^{\prime \prime}}{\left(h_{1} * h_{2}\right)^{\prime}}\right)\right| \\
& =\left|\frac{\left(g_{1} * g_{2}\right)^{\prime}}{\left(h_{1} * h_{2}\right)^{\prime}}+z\left(\frac{\left(h_{1} * h_{2}\right)^{\prime}}{\left[z\left(h_{1} * h_{2}\right)^{\prime}\right]^{\prime}}\right)\left(\frac{\left(g_{1} * g_{2}\right)^{\prime \prime}\left(h_{1} * h_{2}\right)^{\prime}-\left(g_{1} * g_{2}\right)^{\prime}\left(h_{1} * h_{2}\right)^{\prime \prime}}{\left[\left(h_{1} * h_{2}\right)^{\prime}\right]^{2}}\right)\right| \\
& =\left|\frac{\left(g_{1} * g_{2}\right)^{\prime}}{\left(h_{1} * h_{2}\right)^{\prime}}+z \frac{\left(h_{1} * h_{2}\right)^{\prime}}{\left[z\left(h_{1} * h_{2}\right)^{\prime}\right]^{\prime}}\left(\frac{\left(g_{1} * g_{2}\right)^{\prime}}{\left(h_{1} * h_{2}\right)^{\prime}}\right)^{\prime}\right| .
\end{aligned}
$$

Therefore,

$$
\frac{\left(g_{1} * g_{2}\right)^{\prime}}{\left(h_{1} * h_{2}\right)^{\prime}}+z \frac{\left(h_{1} * h_{2}\right)^{\prime}}{\left[z\left(h_{1} * h_{2}\right)^{\prime}\right]^{\prime}}\left(\frac{\left(g_{1} * g_{2}\right)^{\prime}}{\left(h_{1} * h_{2}\right)^{\prime}}\right)^{\prime} \prec z .
$$

On the other hand, since $h_{1} * h_{2} \in \mathscr{K}(0), z\left(h_{1} * h_{2}\right)^{\prime}$ is starlike or

$$
\operatorname{Re}\left\{\frac{z\left[z\left(h_{1} * h_{2}\right)^{\prime}\right]^{\prime}}{z\left(h_{1} * h_{2}\right)^{\prime}}\right\}=\operatorname{Re} \frac{\left[z\left(h_{1} * h_{2}\right)^{\prime}\right]^{\prime}}{\left(h_{1} * h_{2}\right)^{\prime}}>0 .
$$

Thus, in view of Lemma $9,\left(g_{1}(z) * g_{2}(z)\right)^{\prime} /\left(h_{1}(z) * h_{2}(z)\right)^{\prime}<z$ or $\left|G^{\prime}(z) / H^{\prime}(z)\right|=\left|\left[g_{1}(z) * g_{2}(z)\right]^{\prime} /\left[h_{1}(z) * h_{2}(z)\right]^{\prime}\right|<1$.

Remark 10. It is left as an open problem whether Theorem 5(i) can be extended to the case $g_{1}(z)=z^{n} h_{1}(z)$ and $g_{2}^{\prime}(z)=$ $z^{n} h_{2}^{\prime}(z)$ if $n>1$.

\section{Conflicts of Interest}

The authors declare that there are no conflicts of interest regarding the publication of this paper.

\section{References}

[1] J. Clunie and T. Sheil-Small, "Harmonic univalent functions," Annales Academiae Scientiarum Fennicae. Series A. I. Mathematica, vol. 9, pp. 3-25, 1984.

[2] H. Lewy, "On the non-vanishing of the Jacobian in certain one-to-one mappings," Bulletin (New Series) of the American Mathematical Society, vol. 42, no. 10, pp. 689-692, 1936.

[3] D. Bshouty and A. Lyzzaik, "Close-to-convexity criteria for planar harmonic mappings," Complex Analysis and Operator Theory, vol. 5, no. 3, pp. 767-774, 2011.

[4] S. Ruscheweyh and T. Sheil-Small, "Hadamard products of SCHlicht functions and the Polya-SCHoenberg conjecture," Commentarii Mathematici Helvetici, vol. 48, pp. 119-135, 1973.

[5] O. P. Ahuja, J. M. Jahangiri, and H. Silverman, "Convolutions for special classes of harmonic univalent functions," Applied Mathematics Letters, vol. 16, no. 6, pp. 905-909, 2003.

[6] D. Bshouty, S. S. Joshi, and S. B. Joshi, "On close-to-convex harmonic mappings," Complex Variables and Elliptic Equations, vol. 58, no. 9, pp. 1195-1199, 2013.
[7] W. Kaplan, "Close-to-convex schlicht functions," Michigan Mathematical Journal, vol. 1, pp. 169-185, 1952.

[8] S. S. Miller and P. T. Mocanu, "Univalent solutions of BriotBouquet differential equations," Journal of Differential Equations, vol. 56, no. 3, pp. 297-309, 1985.

[9] S. S. Miller and P. T. Mocanu, Differential Subordination-Theory and Applications, vol. 225 of Monorgraphs and Textbooks in Pure and Applied Mathematics, Marcel Dekker, New York, NY, USA, 2000. 


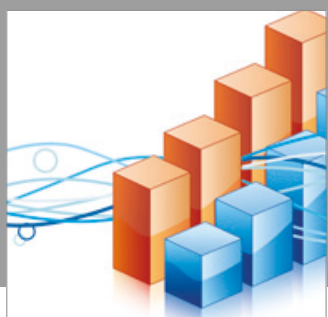

Advances in

Operations Research

vatersals

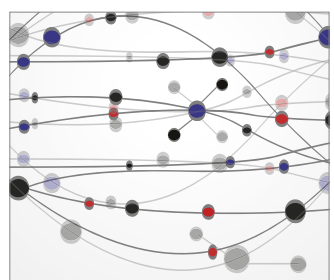

\section{The Scientific} World Journal
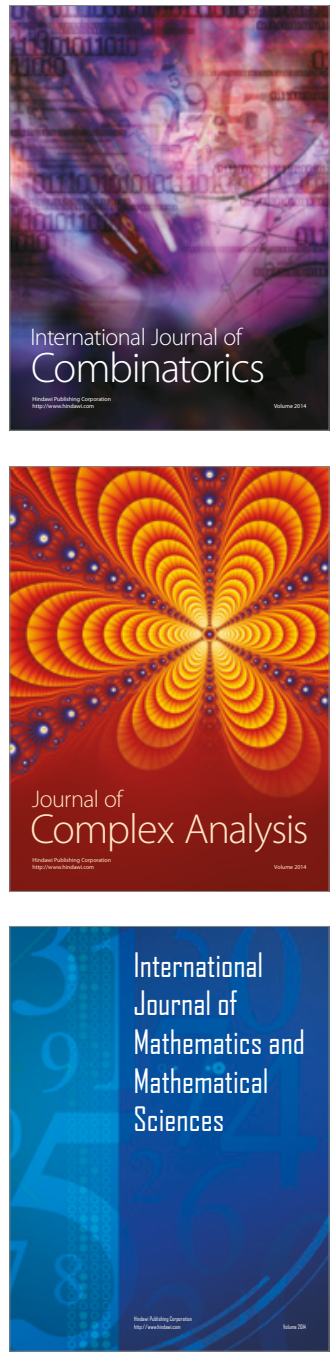
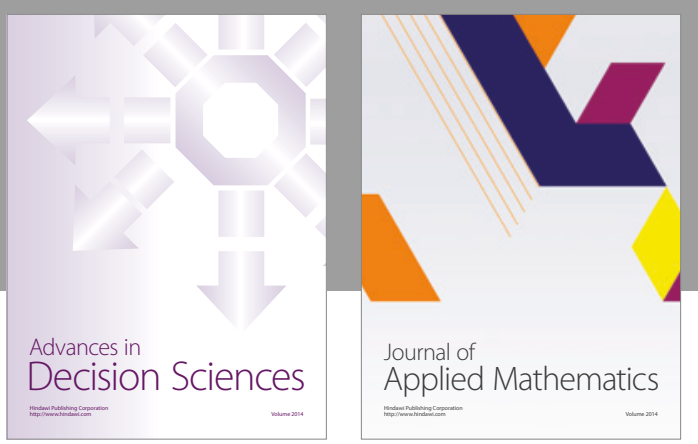

Algebra

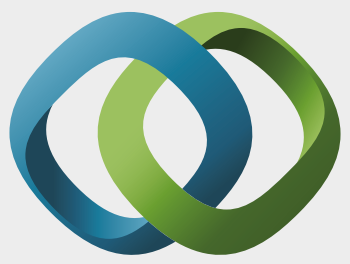

\section{Hindawi}

Submit your manuscripts at

https://www.hindawi.com
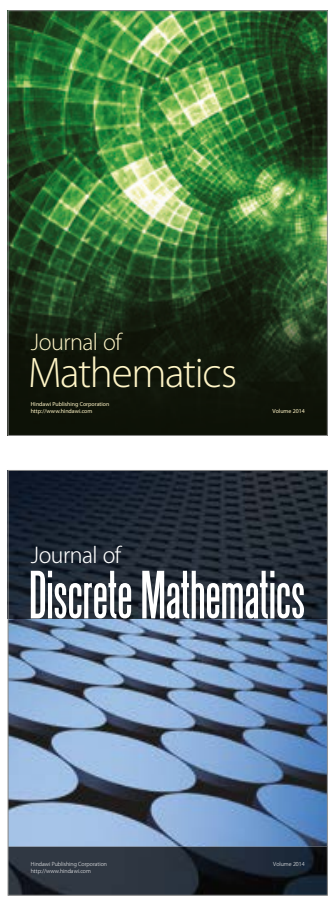

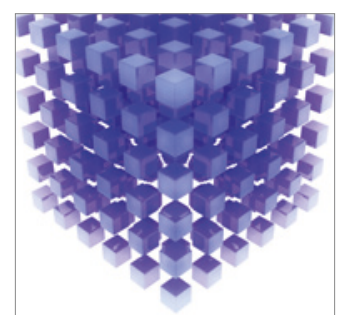

Mathematical Problems in Engineering
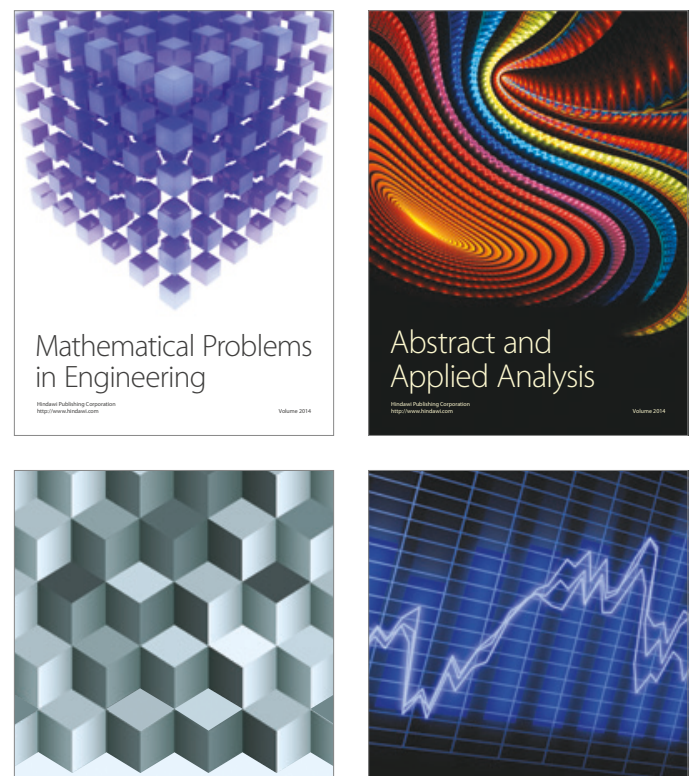

Journal of

Function Spaces

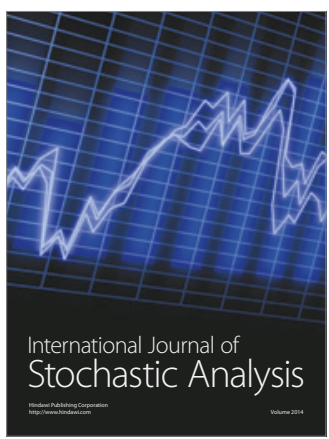

Probability and Statistics
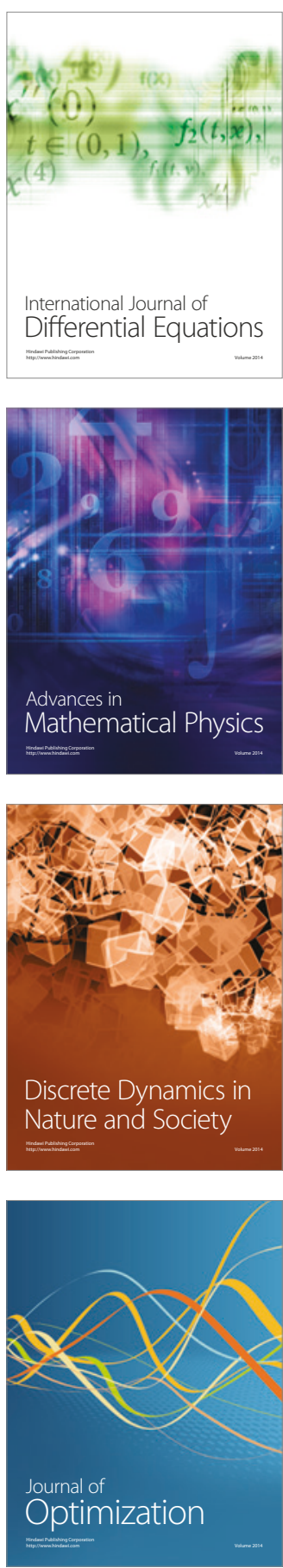